\title{
UNIFORM PARTITIONS OF AN INTERVAL
}

\author{
BY
}

\author{
VLADIMIR DROBOT
}

\begin{abstract}
Let $\left\{x_{n}\right\}$ be a sequence of numbers in $[0,1]$; for each $n$ let $u_{0}(n), \ldots, u_{n}(n)$ be the lengths of the intervals resulting from partitioning of $[0,1]$ by $\left\{x_{1}, x_{2}, \ldots, x_{n}\right\}$. For $p>1$ put $A^{(p)}(n)=(n+1)^{p-1} \Sigma_{0}^{n}\left[u_{j}(n)\right]^{p}$; the paper investigates the behavior of $A^{(p)}(n)$ as $n \rightarrow \infty$ for various sequences $\left\{x_{n}\right\}$. THEOREM 1. If $x_{n}=n \theta(\bmod 1)$ for an irrational $\theta>0$, then $\lim \inf A^{(p)}(n)<\infty$. However $\lim \sup A^{(p)}<\infty$ if and only if the partial quotients of $\theta$ are bounded (in the continued fraction expansion of $\theta$ ). THEOREM 2 gives the exact values for $\lim$ inf and lim sup when $\theta=\frac{1}{2}(1+\sqrt{5})$. THEOREM 3. If $x_{n}$ 's are random variables, uniformly distributed on $[0,1]$, then $\lim A^{(p)}(n)=\Gamma(p+1)$ almost surely.
\end{abstract}

1. Introduction. Let $x_{1}, x_{2}, \ldots$ be an infinite sequence of points between 0 and 1 . For each $n$ the points $x_{1}, x_{2}, \ldots, x_{n}$ partition the interval $[0,1]$ into $n+1$ subintervals. Extensive studies have been made of irregularities of such partitions by considering the quantity $D_{n}$, called discrepancy, defined by

$$
D_{n}=\sup _{0<\alpha<\beta<1}\left|\frac{1}{n} \sum_{j=1}^{n} \chi_{(a, b)}\left(x_{j}\right)-(\beta-\alpha)\right|,
$$

where $\chi_{E}(\cdot)$ is the characteristic function of a set $E$. (See [6].) In this paper we propose to study the problem by introducing a different measure of uniformity defined as follows. For each $n$, let $x_{1}(n), x_{2}(n), \ldots, x_{n}(n)$ be the points $x_{1}, x_{2}, \ldots, x_{n}$ arranged in nondecreasing order, let $x_{0}(n) \equiv 0, x_{n+1}(n) \equiv 1, u_{j}(n)=$ $x_{j+1}(n)-x_{j}(n)(j=0,1, \ldots, n)$, and for each $p>1$ consider

$$
A^{(p)}(n)=(n+1)^{p-1} \sum_{j=0}^{n}\left[u_{j}(u)\right]^{p} .
$$

The closer to 1 the value of $A^{(p)}(n)$ is, the more uniform is the partition (if the points $x_{1}, x_{2}, \ldots, x_{n}$ divide $[0,1]$ into $n+1$ equal parts then $\left.A^{(p)}(n)=1\right)$. In this paper we investigate $\lim _{n} A^{(p)}(n)$ for various sequences $x_{1}, x_{2}, \ldots$ We begin with the classical case $x_{n}=n \theta(\bmod 1)$ for some irrational $\theta>0$. It turns out that the limiting behavior of $A^{(p)}(n)$ strongly depends on the arithmetic character of $\theta$. We have the following theorem.

TheOREM 1. Let $x_{n}=n \theta(\bmod 1)$ for an irrational $\theta>0$ and let $A^{(p)}(n)$ be defined by (1). We have for $p>1$ :

I. $\lim \inf _{n} A^{(p)}(n)<\infty$;

II. $\lim \sup _{n} A^{(p)}(n)<\infty$ if and only if the partial quotients of the continued fraction expansion of $\theta$ are bounded.

Received by the editors June 23, 1980 and, in revised form, September 29, 1980.

AMS (MOS) subject classifications (1970). Primary 10K30. 
For certain values of $\theta$ both $\lim \sup A^{(p)}(n)$ and $\lim \inf A^{(p)}(n)$ can be evaluated as the following theorem shows:

THEOREM 2. Let $\theta=\frac{1}{2}(1+\sqrt{5})$ so that $\theta^{2}=\theta+1$. Let

$$
\psi(t)=5^{-p / 2}(\theta+t)^{p-1}\left[t(\theta-1)^{p}+(1-t) \theta^{p}+t+\theta-1\right] .
$$

Let $A^{(p)}(n)$ be obtained from the sequence $n \theta(\bmod 1)$ as in (1). We have

$$
\begin{gathered}
\lim _{n} \inf A^{(p)}(n)=\psi(0)=\psi(1)=5^{-p / 2}\left(\theta^{2 p-1}+\theta^{p-2}\right), \\
\limsup A_{n}^{(p)}(n)=\psi\left(t_{0}\right),
\end{gathered}
$$

where

$$
t_{0}=\left(1-p^{-1}\right)\left(\theta^{p}-(\theta-1)^{p}-1\right) /\left(\theta^{p}+\theta-1\right)
$$

Special cases of Theorems 1 and $2(p=3)$ appear in [2]. We discuss next the behavior of $A^{(p)}(n)$ in case the sequence $\left\{x_{n}\right\}$ is chosen at random.

THEOREM 3. Let $X_{1}, X_{2}, \ldots$ be a sequence of independent random variables uniformly distributed on $[0,1]$, and let $A^{(p)}(n)$ be the random variable defined by (1). Then $\lim _{n} A^{(p)}(n)=\Gamma(p+1)$ almost surely.

We introduce the following definition.

Definition. Let $\left\{x_{n}\right\}$ be a sequence of numbers in an interval $[0,1]$ and let $A^{(p)}(n)$ be given by (1). We say that this sequence $p$-partitions $[0,1]$ if $\lim _{n} A^{(p)}(n)$ exists.

COROLlaRY. For every $p>1$ there is a sequence which p-partitions $[0,1]$.

This is immediate from Theorem 3. We now proceed with the proofs of the theorems.

2. Proof of Theorems 1 and 2. We summarize first the basic facts about the distribution of the points $\{\theta\},\{2 \theta\}, \ldots,\{n \theta\}$ in $[0,1]$. (Here $\{t\}=t(\bmod 1)$.) For the details and references see [7]. Let $n$ be fixed, let $1 \leqslant a_{n} \leqslant n$ be such that $\left\{a_{n} \theta\right\}$ is the smallest among $\{\theta\},\{2 \theta\}, \ldots,\{n \theta\}$ and let $1 \leqslant b_{n} \leqslant n$ be such that $\left\{b_{n} \theta\right\}$ is the largest. Set $\alpha_{n}=\left\{a_{n} \theta\right\}, \beta_{n}=1-\left\{b_{n} \theta\right\}$. The interval $[0,1]$ is divided by $\{\theta\},\{2 \theta\}, \ldots,\{n \theta\}$ into $n+1$ subintervals as follows: $n+1-a_{n}$ of them are of length $\alpha_{n}, a_{n}+b_{n}-(n+1)$ of them are of length $\alpha_{n}+\beta_{n}$ and $n+1-b_{n}$ have length $\beta_{n}$. The fact that $n+1 \leqslant a_{n}+b_{n}$ can be deduced from the definitions of $a_{n}$ and $b_{n}$. Thus with this notation,

$$
\begin{aligned}
A^{(p)}(n)=(n+1)^{p-1}[ & \left(n+1-a_{n}\right) \alpha_{n}^{p} \\
& \left.+\left(a_{n}+b_{n}-n-1\right)\left(\alpha_{n}+\beta_{n}\right)^{p}+\left(n+1-b_{n}\right) \beta_{n}^{p}\right] .
\end{aligned}
$$

One can actually find $a_{n}$ and $b_{n}$ in terms of the continued fraction expansion of $\theta$ :

$$
\theta=\left[d_{0} ; d_{1}, d_{2}, \ldots\right]=d_{0}+\frac{1}{d_{1}}+\frac{1}{d_{2}}+\cdots
$$


As usual, we set $q_{-1}=0, p_{-1}=1, q_{0}=1, p_{0}=d_{0}, q_{k+1}=d_{k+1} q_{k}+q_{k-1}, p_{k+1}=$ $d_{k+1} p_{k}+p_{k-1}, \delta_{k}=(-1)^{k}\left(q_{k} \theta-p_{k}\right)>0$. Given $n$, to find $a_{n}$ and $\alpha_{n}$ express $n$ as

$$
n=q_{2 m}+r q_{2 m+1}+s, \quad 0 \leqslant r<d_{2 m+2}, 0<s<q_{2 m+1} \text {, }
$$

so that $q_{2 m} \leqslant n<q_{2 m+2}$. Then

$$
a_{n}=q_{2 m}+r q_{2 m+1}, \quad \alpha_{n}=\delta_{2 m}-r \delta_{2 m+1} .
$$

To find $b_{n}$ and $\beta_{n}$ we express $n$ as

$$
n=q_{2 m-1}+u q_{2 m}+v, \quad 0 \leqslant u<d_{2 m+1}, 0 \leqslant v<q_{2 m},
$$

so that $q_{2 m-1} \leqslant n<q_{2 m+1}$. We have

$$
b_{n}=q_{2 m-1}+u q_{2 m}, \quad \beta_{n}=\delta_{2 m-1}-u \delta_{2 m} .
$$

The following are standard facts about continued fractions (see [4]):

$$
\begin{gathered}
d_{k+1}=\left[\delta_{k-1} / \delta_{k}\right], \quad \delta_{k+1}=\delta_{k-1}-d_{k+1} \delta_{k}, \\
d_{k+2} / q_{k+2}<\delta_{k}<1 / q_{k+1} .
\end{gathered}
$$

We are now ready to prove Theorem 1 . Set $x_{n}=n \theta(\bmod 1), p>1$ and let

$$
A(n)=A^{(p)}(n)=(n+1)^{p-1} \sum_{j=0}^{n}\left[u_{j}(n)\right]^{p}
$$

be given as in (1). To show I we will show that $A\left(q_{2 m}+q_{2 m+1}-1\right)$ is bounded. From the discussion above and (2) we see that for $n=q_{2 m}+q_{2 m+1}-1$ the following hold:

$$
a_{n}=q_{2 m}, \quad b_{n}=q_{2 m+1}, \quad \alpha_{n}=\delta_{2 m}, \quad \beta_{n}=\delta_{2 m+1},
$$

and using (7) we get

$$
\begin{aligned}
A(n) & =\left(q_{2 m}+q_{2 m+1}\right)^{p-1}\left(q_{2 m} \delta_{2 m+1}^{p}+q_{2 m+1} \delta_{2 m}^{p}\right) \\
& \leqslant\left(q_{2 m}+q_{2 m+1}\right)^{p-1}\left(q_{2 m} q_{2 m+2}^{-p}+q_{2 m+1} q_{2 m+1}^{-p}\right) \\
& =O(1) \quad(m \rightarrow \infty) .
\end{aligned}
$$

Hence I follows. To show II assume that all the partial quotients $d_{k}$ of $\theta$ are bounded by $D$, say. We wish to show that $A(n)$ is bounded. We claim that a number $c>0$ can be chosen such that the following three inequalities hold:

$$
c^{-1}<\delta_{k-1} / \delta_{k}<c, \quad c^{-1}<a_{n} / b_{n}<c, \quad c^{-1}<\alpha_{n} / \beta_{n}<c .
$$

The first inequality holds for some $c>0$ because $\left[\delta_{k-1} / \delta_{k}\right]=d_{k+1}$ and we are assuming that $d$ 's are bounded. Suppose next that $q_{2 m}<n<q_{2 m+1}$ for some $m$ so that $a_{n}=q_{2 m}$ (see (4)). Let $u$ and $v$ be determined by (5) so that

$$
\begin{aligned}
\frac{a_{n}}{b_{n}} & =\frac{q_{2 m}}{u q_{2 m}+q_{2 m-1}} \leqslant \frac{q_{2 m}}{q_{2 m-1}}=\frac{d_{2 m} q_{2 m-1}+q_{2 m-2}}{q_{2 m-1}} \\
& \leqslant d_{2 m}+1 \leqslant D+1 .
\end{aligned}
$$

On the other hand,

$$
\frac{a_{n}}{b_{n}} \geqslant \frac{q_{2 m}}{d_{2 m+1} q_{2 m}+q_{2 m-1}} \geqslant \frac{1}{d_{2 m+1}+1} \geqslant \frac{1}{D+1} .
$$


If $n$ satisfies $q_{2 m-1} \leqslant n<q_{2 m}$ then the analysis is based on (5) and (3) and leads to the same conclusion. As for the ratio of $\alpha_{n}$ and $\beta_{n}$ we have the following: For $q_{2 m} \leqslant n<q_{2 m+1}$ and $u$ given by (5),

$$
1 \leqslant \frac{\alpha_{n}}{\beta_{n}}=\frac{\delta_{2 m}}{\delta_{2 m-1}-u \delta_{2 m}} \leqslant \frac{\delta_{2 m}}{\delta_{2 m+1}}<d_{2 m+2}+1 \leqslant D+1 .
$$

For $q_{2 m-1} \leqslant n<q_{2 m}$ and $r$ given by (3),

$$
1 \leqslant \frac{\beta_{n}}{\alpha_{n}}=\frac{\delta_{2 m-1}}{\delta_{2 m-2}-r \delta_{2 m-1}} \leqslant \frac{\delta_{2 m-1}}{\delta_{2 m}} \leqslant d_{2 m+1}+1 \leqslant D+1 .
$$

This establishes (8). To show now that $A(n)$ is bounded, we take (2) and bound all the terms by $a_{n} \beta_{n}$ using (8):

$$
\begin{aligned}
A(n) & \leqslant\left(a_{n}+b_{n}\right)^{p-1}\left[b_{n} \alpha_{n}^{p}+\left(b_{n}-1\right)\left(\alpha_{n}+\beta_{n}\right)^{p}+a_{n} \beta_{n}^{p}\right] \\
& \leqslant M\left(a_{n} \beta_{n}\right)^{p}
\end{aligned}
$$

where $M$ depends only on the constant $c$ from (8) (and hence on max $d_{k}$ ). Since $a_{n} \beta_{n}+b_{n} \alpha_{n}=1$, the first part of II follows. We next show the converse of II, that is, if the partial quotients are unbounded then lim $\sup A(n)=\infty$.

There are two cases: either $\left\{d_{2 m}\right\}$ is unbounded or $\left\{d_{2 m+1}\right\}$ is unbounded. We present the arguments in the first case only, the second is completely analogous. For each $m$ let $y_{m}=\left[d_{2 m+2} / 2\right]-2$, so that $y_{m}>0$ for infinitely many $m$ 's and $\lim \sup y_{m}=\infty$. Let

$$
\begin{aligned}
n & =n_{m}=q_{2 m}+\left(y_{m}+1\right) q_{2 m+1}-1 \\
& =q_{2 m+1}+\left(q_{2 m}+y_{m} q_{2 m+1}-1\right) .
\end{aligned}
$$

For those $m$ 's for which $y_{m}>1$ we have from (3)-(6),

$$
\begin{gathered}
a=q_{2 m}+y_{m} q_{2 m+1}, \quad \alpha=\delta_{2 m}-y_{m} \delta_{2 m+1}>y_{m} \delta_{2 m+1}, \\
b=q_{2 m+1}, \quad \beta=\delta_{2 m+1}, \quad a+b=n+1 .
\end{gathered}
$$

Thus, substituting in (2),

$$
\begin{aligned}
A\left(n_{m}\right) & =\left(q_{2 m}+\left(y_{m}+1\right) q_{2 m+1}\right)^{p-1}\left(q_{2 m+1} \alpha^{p}+\left(q_{2 m}+y_{m} q_{2 m+1}\right) \beta^{p}\right) \\
& \geqslant\left(y_{m} q_{2 m+1}\right)^{p-1}\left(q_{2 m+1} y_{m}^{p} \delta_{2 m+1}^{p}\right)=y_{m}^{2 p-1} q_{2 m+1}^{p} \delta_{2 m+1}^{p} .
\end{aligned}
$$

It follows from (7) that

$$
\begin{aligned}
\left(q_{2 m+1} \delta_{2 m+1}\right)^{p} & \geqslant\left[\frac{q_{2 m+1} d_{2 m+3}}{d_{2 m+3} q_{2 m+2}+q_{2 m+1}}\right]^{p} \geqslant\left[\frac{q_{2 m+1}}{q_{2 m+2}+q_{2 m+1}}\right]^{p} \\
& =\left[\frac{q_{2 m+1}}{d_{2 m+2} q_{2 m+1}+q_{2 m}+q_{2 m+1}}\right]^{p}>\left(d_{2 m+2}+3\right)^{-p} .
\end{aligned}
$$

Thus $A\left(n_{m}\right) \geqslant y_{m}^{2 p-1}\left(d_{2 m+2}+3\right)^{-p}$, so lim sup $A(n)=\infty$.

This completes the proof of Theorem 1 and we take up Theorem 2. For $\theta=\frac{1}{2}(1+\sqrt{5})$ all partial quotients are equal to 1 and we have

$$
\begin{aligned}
& q_{-1}=0, \quad q_{0}=1, \quad q_{k+1}=q_{k}+q_{k-1}, \\
& p_{-1}=1, \quad p_{0}=1, \quad p_{k+1}=p_{k}+p_{k-1}, \\
& \delta_{k+1}=\delta_{k-1}-\delta_{k}, \quad q_{k-1}=5^{-1 / 2}\left(\theta^{k}-(-1)^{k} \theta^{-k}\right), \quad \delta_{k}=\theta^{-(k+1)},
\end{aligned}
$$


so that

$$
\lim _{k}\left(q_{k} / \theta^{k}\right)=\theta / \sqrt{5}
$$

Let $0 \leqslant t \leqslant 1$ be given, and suppose $0<t_{k}<1$ are such that $t_{k} q_{2 k-1}-1$ is a positive integer and $t_{k} \rightarrow t$. We will show that if $n_{k}=q_{2 k}+t_{k} q_{2 k-1}-1$ then

$$
\begin{aligned}
\lim _{k} A\left(n_{k}\right) & =5^{-p / 2}(\theta+t)^{p-1}\left[t(\theta-1)^{p}+(1-t) \theta^{p}+t+\theta-1\right] \\
& =\psi(t) .
\end{aligned}
$$

Similarly, if $0<s_{k}<1$ is such that $s_{k} q_{2 k}-1$ is a positive integer and $s_{k} \rightarrow t$, then with $n_{k}=q_{2 k+1}+s_{k} q_{2 k}-1$,

$$
\lim _{k} A\left(n_{k}\right)=\psi(t)
$$

To show (11) we have from (3)-(6):

$$
\begin{aligned}
& a=q_{2 k}, \quad \alpha=\delta_{2 k}, \quad b=q_{2 k-1}, \quad \beta=\delta_{2 k-1}, \quad n+1-a=t_{k} q_{2 k-1}, \\
& a+b-(n+1)=\left(1-t_{k}\right) q_{2 k-1}, \quad n+1-b=q_{2 k}+\left(t_{k}-1\right) q_{2 k-1} .
\end{aligned}
$$

Substituting these values into (2) we get

$$
\begin{aligned}
A\left(n_{k}\right)=\left(q_{2 k}+t_{k} q_{2 k-1}\right)^{p-1}\left[t_{k} q_{2 k} \delta_{2 k}^{p}+\left(1-t_{k}\right) q_{2 k-1}\left(\delta_{2 k}+\delta_{2 k-1}\right)^{p}\right. \\
\left.+\left(q_{2 k}+\left(t_{k}-1\right) q_{2 k-1}\right) \delta_{2 k-1}^{p}\right] .
\end{aligned}
$$

Substituting values for $\delta$ 's from (9) we obtain

$$
\begin{aligned}
A\left(n_{k}\right)=\left(q_{2 k}+t_{k} q_{2 k-1}\right)^{p-1}\left[t_{k} q_{2 k-1} \theta^{-(2 k p+p)}+(1\right. & \left.-t_{k}\right) q_{2 k-1}\left(1+\theta^{-1}\right)^{p} \theta^{-2 k p} \\
& \left.+\left(q_{2 k}+\left(t_{k}-1\right) q_{2 k-1}\right) \theta^{-2 k p}\right] .
\end{aligned}
$$

From (10) it follows then that

$$
\lim _{k} A\left(n_{k}\right)=5^{-p / 2}(\theta+t)^{p-1}\left[t / \theta^{p}+(1-t)\left(1+\theta^{-1}\right)^{p}+\theta+t-1\right],
$$

which implies (11) since $1 / \theta=\theta-1$ and $1+1 / \theta=\theta$. Equation (12) follows pretty much the same way. Let now $\left\{n_{j}\right\}$ be such that $A\left(n_{j}\right)$ converges to $\xi$, say. Clearly $n_{j}$ belongs infinitely often to an interval of the form $\left[q_{2 k+1}, q_{2 k+1}\right)$ or infinitely often to an interval of the form $\left[q_{2 k+1}, q_{2 k+2}\right)$. In the first case $n_{j}=q_{2 k}+$ $t_{k} q_{2 k-1}-1$ for some $0<t_{k}<1$ and $k=k(j)$, depending on $j$; in the second case $n_{j}=q_{2 k+1}+s_{k} q_{2 k}-1,0<s_{k}<1, k=k(j)$. By taking subsequences, if needed, we may assume that $t_{k}$ (or $s_{k}$ ) converges to $t$, say. Thus in both cases $\lim _{j} A\left(n_{j}\right)=\xi$ $=\psi(t)$ for some $0 \leqslant t \leqslant 1$. Hence $\lim \sup A(n)$ and $\lim \inf A(n)$ are, respectively, the maximum and the minimum of $\psi(t)$ for $0 \leqslant t \leqslant 1$. By direct calculation we can obtain that

$$
\psi(0)=\psi(1)=5^{-p / 2}\left(\theta^{2 p-1}+\theta^{p-2}\right) .
$$

The simplification is based on the fact that $\theta^{2}=\theta+1$. Also

$$
\begin{aligned}
5^{p / 2} \psi^{\prime}(t)= & (d / d t)(\theta+t)^{p-1}\left[t\left(-\theta^{p}+(\theta-1)^{p}+1\right)+\theta^{p}+\theta-1\right] \\
= & (d / d t)(\theta+t)^{p-1}[E t+f] \\
= & (\theta+t)^{p-2}[(p-1)(E t+F)+E(\theta+t)] \\
& E=-\theta^{p}+(\theta-1)^{p}+1, \quad F=\theta^{p}+\theta-1 .
\end{aligned}
$$


Solving the equation $\psi^{\prime}(t)=0$ gives the only solution between 0 and 1 :

$$
t_{0}=\left(1-p^{-1}\right)\left(\theta^{p}-(\theta-1)^{p}-1\right) /\left(\theta^{p}+\theta-1\right) .
$$

To finish the proof we will show that $\psi^{\prime}(0)>0$, which is certainly sufficient since $\psi(0)=\psi(1)$ Now, from (14),

$$
\begin{aligned}
5^{p / 2} \psi^{\prime}(0) & =\theta^{p-2}\left[(p-1)\left(\theta^{p}+\theta-1\right)+\theta\left(-\theta^{p}+(\theta-1)^{p}+1\right)\right] \\
& =\theta^{p-2} f(p) .
\end{aligned}
$$

Thus it is enough to show that $f(p)>0$ for $p>1$. Direct calculation gives $f(1)=0$ and

$$
\begin{aligned}
f^{\prime}(p)= & (p-1) \theta^{p} \log \theta+\theta^{p}+\theta-1 \\
& +\theta\left[-\theta^{p} \log \theta+(\theta-1)^{p} \log (\theta-1)\right] \\
= & \theta^{p}[(p-1) \log \theta+1-\theta \log \theta]-(\log \theta) / \theta^{p-1}+\theta-1 .
\end{aligned}
$$

Since $1-\theta \log \theta=0.221 \ldots, f^{\prime}(p)$ is an increasing function for $p>1$. Also,

$$
f^{\prime}(1)=\theta(1-\theta \log \theta)-\log \theta+\theta-1=0.495 \ldots
$$

so that $f(p)$ is positive for $p>1$. The proof of Theorem 2 is now complete.

3. Proof of Theorem 3. Since $p$ is going to be fixed throughout, we will write $A_{n}$ for $A^{(p)}(n)$. The basic tool to be used is the martingale convergence theorem: Let $F_{n}$ be an increasing sequence of $\sigma$-fields, $Z_{n}$ a random variable measurable with respect to $F_{n}$. If $E\left(Z_{n+1} \mid F_{n}\right)=Z_{n}$ and $\sup _{n} E\left(\left|Z_{n}\right|\right)<\infty$, then the sequence $Z_{n}$ converges almost surely. $E(Z \mid F)$ is the conditional expectation of $Z$ relative to $F$. (See J. L. Doob [1] for the details.) We let $\left(X_{1}^{(n)}, \ldots, X_{n}^{(n)}\right)$ be the order statistic of size $n$, i.e. the values of $X_{1}, X_{2}, \ldots, X_{n}$ arranged in increasing order, put $X_{0}^{(n)} \equiv 0$, $X_{n+1}^{(n)} \equiv 1$ and introduce random variables $U_{j}(n)=X_{j+1}^{(n)}-X_{j}^{(n)}$ so that once again

$$
A_{n}=(n+1)^{p-1} \sum_{j=0}^{n}\left[U_{j}(n)\right]^{p}
$$

is a random variable. We take our $\sigma$-fields to be $F_{n}=F\left(U_{0}, U_{1}, \ldots, U_{n}\right)$, the $\sigma$ fields generated by the random variables $U_{0}, U_{1}, \ldots, U_{n}$ and consider the random variable

$$
Z_{n}=A_{n}+\sum_{j=1}^{n-1}\left[A_{j}-E\left(A_{j+1} \mid F_{j}\right)\right] .
$$

We will show the following:

(a) $\left\{Z_{n}\right\}$ is a martingale relative to $\left\{F_{n}\right\}$.

(b) $\sum_{n=1}^{\infty} E\left(\left|A_{n}-E\left(A_{n+1} \mid F_{n}\right)\right|\right)<\infty$.

(c) $\lim _{n} E\left(\left|A_{n}\right|\right)=\Gamma(p+1)$.

Note that (b), (c) imply $\lim \sup E\left(\left|Z_{n}\right|\right)<\infty$ so that $Z_{n}$ converges a.e. by the martingale convergence theorem. In addition, (b) shows $\sum\left[A_{n}-E\left(A_{n+1} \mid F_{n}\right)\right]$ converges a.e. and thus $A_{n}$ converges a.e. The limit will be identified later. The proof of (a) is straightforward:

so that $E\left(Z_{n+1} \mid F_{n}\right)=Z_{n}$.

$$
\begin{aligned}
Z_{n+1} & =Z_{n}+A_{n+1}-A_{n}+A_{n}-E\left(A_{n+1} \mid F_{n}\right) \\
& =Z_{n}+A_{n+1}-E\left(A_{n+1} \mid F_{n}\right)
\end{aligned}
$$


Before we take up (b) we recall facts regarding random variables $U_{0}(n), U_{1}(n), \ldots, U_{n}(n)$ (see [5, Chapter 9]). Since $U_{0}(n)+\cdots+U_{n}(n) \equiv 1$, the $U$ 's are certainly not independent, but if we delete one of them, the remaining $n$ are "uniformly" distributed on the simplex

$$
T_{n}=\left\{\left(t_{1}, t_{2}, \ldots, t_{n}\right): t_{j} \geqslant 0, t_{1}+t_{2}+\cdots+t_{n}<1\right\}
$$

more precisely, the joint density function of the remaining $n$ is given by

$$
f_{n}\left(t_{1}, t_{2}, \ldots, t_{n}\right)= \begin{cases}n ! & \text { if }\left(t_{1}, t_{2}, \ldots, t_{n}\right) \in T_{n}, \\ 0 & \text { otherwise. }\end{cases}
$$

Thus for any $\alpha>0$ and any $i$,

$$
E\left(\left[U_{i}(n)\right]^{\alpha}\right)=n ! \int_{T_{n}} x_{i}^{\alpha} d x_{1} d x_{2} \ldots d x_{n}=\frac{n ! \Gamma(\alpha+1)}{\Gamma(n+\alpha+1)} .
$$

Similarly for any $\alpha>0, \beta>0, i \neq j$,

$$
\begin{aligned}
E\left(\left[U_{i}(n)\right]^{\alpha}\left[U_{j}(n)\right]^{\beta}\right) & =n ! \int_{T_{n}} x_{i}^{\alpha} x_{j}^{\beta} d x_{1} \ldots d x_{n} \\
& =n ! \Gamma(\alpha+1) \Gamma(\beta+1) / \Gamma(n+\alpha+\beta+1) .
\end{aligned}
$$

The values of these integrals can be either evaluated directly or looked up in [3]. Notice that the right-hand side of both of the above formulas is independent of $i$ and $j$.

To prove (b) we use the Cauchy-Schwarz inequality $E(|W|)<\left[E\left(W^{2}\right)\right]^{1 / 2}$ with $W=E\left(A_{n+1} \mid F_{n}\right)-A_{n}$ and show that for some constant $c(p)$, depending only on $p$, we have

$$
E\left(\left[E\left(A_{n+1} \mid F_{n}\right)-A_{n}\right]^{2}\right)<c(p) n^{-3} \text {. }
$$

This will certainly prove (b) since $\Sigma n^{-3 / 2}$ converges. We derive now the formula for $E\left(A_{n+1} \mid F_{n}\right)$. Since $X_{n+1}$ is uniformly distributed on $[0,1]$ and independent of $U_{0}, \ldots, U_{n}$ we have

$$
\begin{aligned}
E\left(A_{n+1} \mid\right. & \left.U_{0}(n)=u_{0}, U_{1}(n)=u_{1}, \ldots, U_{n}(n)=u_{n}\right) \\
= & (n+2)^{p-1} \sum_{j=0}^{n} \int_{0}^{u_{j}}\left[\sum_{\substack{i=0 \\
i \neq j}}^{n} u_{i}^{p}+t^{p}+\left(u_{j}-t\right)^{p}\right] d t \\
= & (n+2)^{p-1} \sum_{j=0}^{n}\left[\sum_{\substack{i=0 \\
i \neq j}}^{n}\left(u_{i}^{p} u_{j}\right)+\frac{2}{p+1} u_{j}^{p+1}\right] \\
= & (n+2)^{p-1}\left[\frac{1}{(n+1)^{p-1}} A_{n}-\sum_{j=0}^{n}\left(1-\frac{2}{p+1}\right) u_{j}^{p+1}\right] \\
= & A_{n}+\left[\frac{(n+2)^{p-1}-(n+1)^{p-1}}{(n+1)^{p-1}}\right] A_{n}-(n+2)^{p-1} \frac{p-1}{p+1} \sum_{j=0}^{n} u_{j}^{p+1} \\
= & A_{n}+\left[(n+2)^{p-1}-(n+1)^{p-1}\right] \sum_{j=0}^{n} u_{j}^{p}-(n+2)^{p-1} \frac{p-1}{p+1} \sum_{j=0}^{n} u_{j}^{p+1} .
\end{aligned}
$$


Thus

$$
E\left(A_{n+1} \mid F_{n}\right)-A_{n}=\sum_{j=0}^{n}\left[a_{n} U_{j}^{p}(n)-b_{n} U_{j}^{p+1}(n)\right]
$$

where

$$
a_{n}=(n+2)^{p-1}-(n+1)^{p-1}=(p-1) n^{p-2}(1+o(1))
$$

and

$$
b_{n}=(n+2)^{p-1} \frac{p-1}{p+2}=n^{p-1} \frac{p-1}{p+1}(1+o(1)) .
$$

In view of remarks after (16) we get (writing $U_{k}$ for $U_{k}(n)$ )

$$
\begin{aligned}
& E\left(\left[E\left(A_{n+1} \mid F_{n}\right)-A_{n}\right]^{2}\right)=E\left(\left[\sum_{j=0}^{n} a_{n} U_{j}^{p}-b_{n} U_{j}^{p+1}\right]^{2}\right) \\
&=n E\left(\left[a_{n} U_{0}^{p}-b_{n} U_{0}^{p+1}\right]^{2}\right) \\
& \quad+n(n+1) E\left(\left[a_{n} U_{0}^{p}-b_{n} U_{0}^{p+1}\right]\left[a_{n} U_{1}^{p}-b_{n} U_{1}^{p+1}\right]\right) \\
&=n\left[a_{n}^{2} E\left(U_{0}^{2 p}\right)-2 a_{n} b_{n} E\left(U_{0}^{2 p+1}\right)+b_{n} E\left(U_{0}^{2 p+2}\right)\right] \\
&+n(n+1)\left[a_{n}^{2} E\left(U_{0}^{p} U_{1}^{p}\right)-a_{n} b_{n}\left\{E\left(U_{0}^{p} U_{1}^{p+1}\right)+E\left(U_{0}^{p+1} U_{1}^{p}\right)\right\}\right. \\
&\left.+b_{n}^{2} E\left(U_{0}^{p+1} U_{1}^{p+1}\right)\right] \\
&= n P_{n}+n(n+1) Q_{n} .
\end{aligned}
$$

We will show that both $n P_{n}$ and $n(n+1) Q_{n}$ are $O\left(n^{-3}\right)$, the implicit constant depending on $p$ only. Before we do that we need the following estimate:

$$
n ! / \Gamma(n+2 p+1) \leqslant C_{1}(p) n^{-2 p}
$$

$C_{1}(p)$ depending on $p$ alone. Indeed, using Stirling's formula

$$
\log \Gamma(x)=\left(x-\frac{1}{2}\right) \log x-x+\log \sqrt{2 \pi}+o(1)
$$

we get

$$
\begin{aligned}
& \log (n ! / \Gamma(n+2 p+1))=\log \Gamma(n+1)-\log \Gamma(n+2 p+1) \\
&=\left(n+\frac{1}{2}\right) \log (n+1)-(n+1)-\left(n+2 p+\frac{1}{2}\right) \log (n+2 p+1) \\
&+n+2 p+1+O(1) \\
&= n[\log (n+1)-\log (n+2 p+1)] \\
&+\frac{1}{2}[\log (n+1)-\log (n+2 p+1)]-2 p \log n+O(1)
\end{aligned}
$$

Since for any fixed $d, x(\log (x+d)-\log x) \rightarrow d(x \rightarrow \infty)$, the result follows. We now estimate $n P_{n}$. From the definition of $P_{n}$ and (15)-(17) we have

$$
\begin{aligned}
n P_{n}=n ! n[(p & -1)^{2} n^{2 p-4} \frac{\Gamma(2 p+1)}{\Gamma(n+2 p+1)}-\frac{(p-1)^{2}}{p+1} n^{2 p-3} \\
& \left.\quad \times \frac{\Gamma(2 p+2)}{\Gamma(n+2 p+2)}+\left(\frac{p-1}{p+1}\right)^{2} n^{2 p-2} \frac{\Gamma(2 p+3)}{\Gamma(n+2 p+3)}\right](1+o(1)) .
\end{aligned}
$$


Using the identity $x \Gamma(x)=\Gamma(x+1)$ several times we get

$$
\begin{aligned}
n P_{n} & =\frac{n^{2 p-3}(p-1)^{2} n !}{\Gamma(n+2 p+1)} \Gamma(2 p+1) \\
& \times\left[1-\frac{2 p+1}{p+1} \frac{n}{n+2 p+1}+\frac{(2 p+1) n^{2}}{(p+1)(n+2 p+1)(n+2 p+2)}\right](1+o(1)),
\end{aligned}
$$

so the estimate $n P_{n}=O\left(n^{-3}\right)$ follows from (18). Next we estimate $n(n+1) Q_{n}$, again using (15)-(17):

$$
\begin{aligned}
n(n+1) Q_{n}=n^{2} n ! & (p-1)^{2} n^{2 p-4} \frac{\Gamma^{2}(p+1)}{\Gamma(n+2 p+1)} \\
& -2 \frac{(p-1)^{2}}{p+1} n^{2 p-3} \frac{\Gamma(p+1) \Gamma(p+2)}{\Gamma(n+2 p+2)} \\
& \left.+\left(\frac{p-1}{p+1}\right)^{2} n^{2 p-2} \frac{\Gamma^{2}(p+2)}{\Gamma(n+2 p+3)}\right](1+o(1)) \\
= & \frac{n ! n^{2 p-2}(p-1)^{2} \Gamma^{2}(p+1)}{\Gamma(n+2 p+1)} \\
& \times\left[1-\frac{2 n}{2 p+n+1}+\frac{n^{2}}{(n+2 p+1)(n+2 p+2)}\right](1+o(1)) .
\end{aligned}
$$

The expression in square brackets is equal to

$$
(-n+(2 p+1)(2 p+2)) /(n+2 p+1)(n+2 p+2)=O\left(n^{-1}\right) .
$$

Hence it follows from (18) that

$$
n(n+1) Q_{n}=C(p) \frac{n ! n^{2 p-3}}{\Gamma(n+2 p+1)}(1+o(1))=O\left(n^{-3}\right) .
$$

Thus (b) is proved.

To show (c) we evaluate $E\left(A_{n}\right)$ directly from (15):

$$
E\left(A_{n}\right)=(n+1)^{p-1} \sum_{j=0}^{n} E\left(U_{n}^{p}(n)\right)=\frac{(n+1)^{p-1} n n ! \Gamma(p+1)}{\Gamma(n+p+1)} .
$$

We show now that $\gamma_{n}=(n+1)^{p-1} n n ! / \Gamma(n+p+1) \rightarrow 1(n \rightarrow \infty)$ : Clearly $\gamma_{n} \sim$ $\beta_{n}=n^{p} n ! / \Gamma(n+p+1)$, so using Stirling's formula,

$$
\begin{aligned}
\log \beta_{n}= & p \log n+\left(n+\frac{1}{2}\right) \log (n+1)-(n+1)+\frac{1}{2} \log (2 \pi) \\
& -\left(n+p+\frac{1}{2}\right) \log (n+p+1)+n+p+1-\frac{1}{2} \log (2 \pi)+o(1) \\
= & n[\log (n+1)-\log (n+1+p)]+p+o(1) .
\end{aligned}
$$

Again, $x[\log (x+d)-\log (x)] \rightarrow d(x \rightarrow \infty)$, so $\log \beta_{n} \rightarrow 0(n \rightarrow \infty)$, proving the assertion. Thus $\lim _{n} E\left(A_{n}\right)=\Gamma(p+1)$. This completes the proof of (a)-(c) and shows that $A_{n}^{(p)}$ converges almost surely. What remains is the identification of the limit. Since $E\left(A_{n}\right) \rightarrow \Gamma(p+1)$ it is reasonable to expect that $A_{n} \rightarrow \Gamma(p+1)$ since 
the limit should be constant a.e. To establish it rigorously we show that $A_{n} \rightarrow$ $\Gamma(p+1)$ in probability. This proof is due to Professor Boris Pittel. Let $Y_{0}, Y_{1}, \ldots$ be a sequence of exponentially distributed independent random variables, so that $P\left(Y_{s}<t\right)=1-e^{-t}$. Let $S_{n}=Y_{0}+Y_{1}+\cdots+Y_{n}$. It is known that the vectors $\left(U_{0}(n), U_{1}(n), \ldots, U_{n}(n)\right)$ and $\left(Y_{0} / S_{n}, Y_{1} / S_{n}, \ldots, Y_{n} / S_{n}\right)$ have the same distribution (see [5, p. 242]). Therefore the distributions of $A_{n}$ and

$$
(n+1)^{p-1}\left[\sum_{j=0}^{n} Y_{j}^{p}\right] / S_{n}^{p}
$$

are also the same. By the strong law of large numbers,

$$
\frac{(n+1)^{p-1} \sum_{j=0}^{n} Y_{j}^{p}}{S_{n}^{p}}=\frac{(n+1)^{-1} \sum_{j=0}^{n} Y_{j}^{p}}{\left(S_{n} / n+1\right)^{p}} \rightarrow \frac{E\left(Y_{0}^{p}\right)}{\left(E\left(Y_{0}\right)\right)^{p}}=\Gamma(p+1)
$$

almost everywhere, and thus in probability. Hence $A_{n}^{(p)}$ also converges to $\Gamma(p+1)$ in probability. The proof of Theorem 3 is thus completed.

\section{REFERENCES}

1. J. L. Doob, Stochastic processes, Wiley, New York, 1953.

2. V. Drobot, Approximation of curves by polygons (to appear).

3. I. S. Gradshtein and I. M. Ryzhik, Tables of integrals, sums and products, Moscow, 1971.

4. G. H. Hardy and E. M. Wright, An introduction to the theory of numbers, Clarendon Press, Oxford, 1960.

5. S. Karlin, A first course in stochastic processes, Academic Press, New York, 1968.

6. L. Kuipers and H. Niederreiter, Uniform distribution of sequences, Wiley, New York, 1974.

7. N. B. Slater, Gaps and steps for the sequence $n \theta \bmod 1$, Proc. Cambridge Philos. Soc. 63 (1967), 1115-1123.

Department of Mathematics, University of Santa Clara, Santa Clara, California 95053 